科 学 通 报

\title{
$\mathrm{C}_{60}$ 分子气相摩尔摘及摩尔热容的理论计算
}

\author{
刘奉岭宁世光 \\ (山东师范大学化学系, 济南 250014)
}

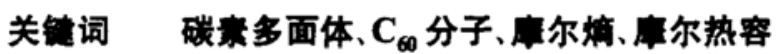

自从 1990 年文献[1] 报道成功的常量制备并分离出稳定存在的 $\mathrm{C}_{60}$ 和 $\mathrm{C}_{70}$ 原子䉈以来, 人 们对这类新型碳素多面体分子的研究寄予了极大的热情和期望, 取得了大量的研究成果. $\mathrm{C}_{60}$ 分子虽有固态时摩尔热容的实验及理论计算结果 ${ }^{\left({ }^{2}\right]}$, 而气相摩尔嫡及摩尔热容的实验和理论 计算结果, 就我们所接触的文献均未见报道. 文献[3] 报道了不同温度所测定的 $\mathrm{C}_{60}$ 分子的蒸 气压, 因而计算 $\mathrm{C}_{60}$ 分子气相摩尔嫡及摩尔热容对于了解该分子的热力学性质有一定实际意 义. 本文采用统计力学方法计算了 $\mathrm{C}_{60}$ 分子气态时的摩尔摘和摩尔热容.

\section{1 计算方法及所用数据}

单个 $C_{60}$ 分子是稳定的具有 $I_{h}$ 对称性的分子, 因而其在气相中有三个平动自由度、三个转 动自由度和 174 个振动自由度. 这 174 个振动模式不可约表示为

$$
\Gamma_{v i b}=2 A_{1 g}+3 T_{1 \mathrm{~g}}+6 G_{g}+8 H_{g}+A_{u}+4 T_{1 u}+5 T_{2 u}+6 G_{\mathrm{u}}+7 H_{u} \text {. }
$$

文献[4] 给出了这 174 个振动频率的具体计算数值.

$\mathrm{C}_{60}$ 分子的气相摩尔嫡及摩尔热容的计算均采用统计力学方法及理想气体模型. 其摩尔 嫡表示为平动嫡 $S_{m}^{t}$ 、转动摘 $S_{m}^{r}$ 和振动摘 $S_{m}^{v}$ 之和, 即有下式 (分子中电子的自旋多重度是 1 忽 略其贡献):

$$
S_{m}=S_{m}^{t}+S_{m}^{r}+S_{m}^{v}
$$

$S_{m}^{t}, S_{m}^{r}$ 及 $S_{m}^{v}$ 的计算分别采用统计力学方法. 计算 $S_{m}^{t}$ 时, 所用的压力为 $101.325 \mathrm{kPa}$; 计算 $S_{m}^{r}$ 时所用转动惯量是根据 $\mathrm{C}_{60}$ 分子结构数据计算求得的, 其数值为 $I_{x}=I_{y}=I_{z}=994.15 \times 10^{-46} \mathrm{~kg} \cdot \mathrm{m}^{2}$; 计算 $S_{m}^{v}$ 时所用振动频率数值取自文献[4] 的计算数值.

$\mathrm{C}_{60}$ 分子气相摩尔热容的计算采用下面的公式:

$$
\begin{gathered}
C_{v, m}=3 R+R \cdot \sum_{i=1}^{174}\left\{\left(\frac{\Theta_{v, i}}{T}\right)^{2} \frac{\exp \left(\Theta_{v, i} / T\right)}{\left[\exp \left(\Theta_{v, i} / T\right)-1\right]^{2}}\right\}, \\
C_{p, m}=C_{v, m}+R,
\end{gathered}
$$

式 (2), (3) 中, $C_{v, m}$ 和 $C_{p, m}$ 分别为摩尔等容热容和摩尔等压热容, $R$ 是气体常数, $T$ 是绝对温 度, $\Theta_{v, i}$ 是第 $i$ 个特征振动温度. 计算 $C_{v, m}$ 和 $C_{p, m}$ 时, 所用振动频率也是取自文献[4].

1993-08-10 收稿, 199401-10 收修改稿. 


\section{2 结 果 及 讨 论}

本文根据式 (1), (2), (3) 分别计算了 $\mathrm{C}_{60}$ 分子在气相中, $101.325 \mathrm{kPa}$ 压力下, 不同温度时 的摩尔平动嫡 $S_{m}^{t}$ 、摩尔转动嫡 $S_{m}^{r}$ 、摩尔振动嫡 $S_{m}^{v}$ 及总摩尔嫡 $S_{m}$ 和摩尔热容, 结果在表 1 中给 出.

表 1 计算的 $C_{60}$ 分子气相摩尔嫡及摩尔热容 ${ }^{\mathrm{a})}$

\begin{tabular}{|c|c|c|c|c|c|c|}
\hline$T(\mathrm{~K})$ & $S_{m}^{t}$ & $S_{m}^{r}$ & $S_{m}^{0}$ & $S_{m}$ & $C_{v, \mathrm{~m}}$ & $C_{P . m}$ \\
\hline 200 & 182.51 & 117.97 & 103.87 & 404.36 & 272.64 & 280.96 \\
\hline 250 & 187.15 & 120.75 & 171.17 & 479.08 & 384.40 & 392.72 \\
\hline 300 & 190.94 & 123.03 & 246.39 & 560.36 & 493.03 & 501.35 \\
\hline 350 & 194.14 & 124.95 & 326.31 & 645.41 & 595.22 & 603.53 \\
\hline 400 & 196.91 & 126.62 & 408.69 & 732.23 & 689.09 & 697.40 \\
\hline 450 & 199.36 & 128.08 & 491.90 & 819.35 & 773.77 & 782.08 \\
\hline 500 & 201.55 & 129.40 & 574.78 & 905.74 & 849.13 & 857.45 \\
\hline 550 & 203.53 & 130.59 & 656.51 & 990.65 & 915.60 & 923.91 \\
\hline 600 & 205.34 & 131.67 & 736.57 & 1073.50 & 973.89 & 982.20 \\
\hline 650 & 207.01 & 132.67 & 814.58 & 1154.27 & 1024.86 & 1033.17 \\
\hline 700 & 208.55 & 133.60 & 890.35 & 1232.50 & 1069.39 & 1077.71 \\
\hline 750 & 209.98 & 134.46 & 963.77 & 1308.21 & 1108.32 & 1116.63 \\
\hline 800 & 211.32 & 135.26 & 1034.80 & 1381.39 & 1142.40 & 1150.72 \\
\hline 850 & 212.58 & 136.02 & 1103.46 & 1452.07 & 1172.31 & 1180.62 \\
\hline 900 & 213.77 & 136.73 & 1169.81 & 1520.32 & 1198.62 & 1206.94 \\
\hline 950 & 214.89 & 137.40 & 1233.90 & 1586.21 & 1221.85 & 1230.16 \\
\hline 1000 & 215.96 & 138.04 & 1295.83 & 1649.84 & 1242.40 & 1250.72 \\
\hline 1050 & 216.98 & 138.65 & 1355.68 & 1711.31 & 1260.66 & 1268.98 \\
\hline 1100 & 217.94 & 139.23 & 1413.55 & 1770.73 & 1276.93 & 1285.25 \\
\hline 1150 & 218.87 & 139.79 & 1469.53 & 1828.19 & 1291.47 & 1299.79 \\
\hline 1200 & 219.75 & 140.32 & 1523.71 & 1883.79 & 1304.51 & 1312.82 \\
\hline 1250 & 220.60 & 140.83 & 1576.19 & 1937.62 & 1316.23 & 1324.55 \\
\hline 1300 & 221.41 & 141.32 & 1627.04 & 1989.78 & 1326.81 & 1335.13 \\
\hline 1350 & 222.20 & 141.79 & 1676.36 & 2040.35 & 1336.38 & 1344.69 \\
\hline 1400 & 222.96 & 142.24 & 1724.21 & 2089.41 & 1345.06 & 1353.37 \\
\hline 1450 & 223.68 & 142.68 & 1770.67 & 2137.04 & 1352.95 & 1361.26 \\
\hline 1500 & 224.39 & 143.10 & 1815.82 & 2183.32 & 1360.15 & 1368.46 \\
\hline 1550 & 225.07 & 143.51 & 1859.71 & 2228.30 & 1366.72 & 1375.04 \\
\hline 1600 & 225.73 & 143.91 & 1902.41 & 2272.05 & 1372.75 & 1381.06 \\
\hline 1650 & 226.37 & 144.29 & 1943.97 & 2314.03 & 1378.28 & 1386.59 \\
\hline 1700 & 226.99 & 144.66 & 1984.44 & 2356.10 & 1383.37 & 1391.68 \\
\hline 1750 & 227.59 & 145.02 & 2023.89 & 2396,51 & 1388.06 & 1396.38 \\
\hline 1800 & 228.18 & 145.37 & 2062.35 & 2435.91 & 1392.40 & 1400.71 \\
\hline 1850 & 228.75 & 145.72 & 2099.87 & 2474.35 & 1396.41 & 1404.72 \\
\hline 1900 & 229.30 & 146.05 & 2136.50 & 2511.86 & 1400.13 & 1408.44 \\
\hline 1950 & 229.84 & 146.37 & 2172.26 & 2548.49 & 1403.58 & 1411.90 \\
\hline 2000 & 230.37 & 146.69 & 2207.21 & 2548.28 & 1406.80 & 1415.11 \\
\hline 2050 & 230.88 & 147.00 & 2241.37 & 2619.26 & 1409.79 & 1418.11 \\
\hline 2100 & 231.38 & 147.30 & 2274.77 & 2653.46 & 1412.59 & 1420.90 \\
\hline 2150 & 231.87 & 147.59 & 2307.46 & 2686.93 & 1415.20 & 1423.51 \\
\hline 2200 & 232.35 & 147.88 & 2339.45 & 2719.68 & 1417.64 & 1425.96 \\
\hline 2250 & 232.82 & 148.16 & 2370.77 & 2751.75 & 1419.94 & 1428.25 \\
\hline 2300 & 233.27 & 148.43 & 2401.45 & 2783.17 & 1422.09 & 1430.40 \\
\hline 2350 & 233.72 & 148.70 & 2431.52 & 2813.95 & 1424.11 & 1432.42 \\
\hline 2400 & 234.16 & 148.96 & 2461.00 & 2844.13 & 1426.01 & 1434.32 \\
\hline 2450 & 234.59 & 149.22 & 2489.91 & 2873.72 & 1427.80 & 1436.11 \\
\hline 2500 & 235.01 & 149.47 & 2518.27 & 2902.76 & 1429.48 & 1437.80 \\
\hline 2550 & 235.42 & 149.72 & 2546.10 & 2931.24 & 1431.08 & 1439.39 \\
\hline 2600 & 235.82 & 149.96 & 2573.42 & 2959.21 & 1432.58 & 1440.90 \\
\hline 2650 & 236.22 & 150.20 & 2600.24 & 2986.67 & 1434.00 & 1442.32 \\
\hline
\end{tabular}


表 1 (续)

\begin{tabular}{ccccccc}
\hline$T(\mathrm{~K})$ & $S_{m}^{t}$ & $S_{m}^{r}$ & $S_{m}^{v}$ & $S_{m}$ & $C_{\text {o.m }}$ & $C_{P, m}$ \\
\hline 2700 & 150.43 & 169.58 & 2626.59 & 3013.64 & 1435.35 & 1443.67 \\
2750 & 150.66 & 169.80 & 2652.49 & 3040.14 & 1436.63 & 1444.94 \\
2800 & 150.88 & 170.03 & 2677.93 & 3066.19 & 1437.84 & 1446.16 \\
2850 & 151.11 & 170.25 & 2702.95 & 3091.80 & 1438.99 & 1447.31 \\
2900 & 151.32 & 170.47 & 2727.55 & 3116.98 & 1440.08 & 1448.40 \\
2950 & 151.54 & 170.68 & 2751.75 & 3141.75 & 1441.12 & 1449.44 \\
3000 & 151.75 & 170.89 & 2775.56 & 3166.12 & 1442.11 & 1450.43 \\
\hline
\end{tabular}

a) 计算平动嫡时所用压力为 $101325 \mathrm{~Pa}$; 本表中嫡的单位是 $\mathrm{J} \cdot \mathrm{mol}^{-1} \cdot \mathrm{K}^{-1}$, 热容的单位也是 $\mathrm{J} \cdot \mathrm{mol}^{-1} \cdot \mathrm{K}^{-1}$.

由表 1 可见, 平动嫡 $S_{m}^{t}$ 和转动摘 $S_{m}^{r}$ 随温度变化很小, 而振动摘 $S_{m}^{v}$ 随温度升高增加得很 快. 这说明低温时大多数处于振动能级的基态, 随着温度的升高处于振动激发态数目逐渐增 高. 总的摩尔嫡 $S_{m}$ 随温度的变化主要是振动嫡 $S_{m}^{v}$ 变化引起的. 由于平动嫡 $S_{m}^{t}$ 和转动嫡 $S_{m}^{r}$ 随温度变化很小, 从表 1 可见高温时振动嫡 $S_{m}^{v}$ 是摩尔嫡 $S_{m}$ 的主要部分, 这可从 $\mathrm{C}_{60}$ 分子具有 较多 (174 个) 振动模式加以解释. 为了得到摩尔嫡 $S_{m}$ 随温度的变化关系, 将表 1 中摩尔嫡 $S_{m}$ 对热力学温度 $T(\mathrm{~K})$ 作回归分析, 同时考虑压力 $P($ 单位是 $\mathrm{Pa})$ 的影响, 得到计算 $\mathrm{C}_{60}$ 分子气相 摩尔摘 $S_{m}$ 的公式, 即 $\left(S_{m}\right.$ 的单位 $\left.\mathrm{J} \cdot \mathrm{mol}^{-1} \cdot \mathrm{K}^{-1}\right)$ :

$$
\begin{gathered}
S_{m}=51.679+1.654 \cdot T+3.802 \times 10^{-4} \cdot T^{2}-6.471 \times 10^{-7} \cdot T^{3}, \\
+2.380 \times 10^{-10} \cdot T^{4}-2.913 \times 10^{-14} \cdot T^{5}-8.314 \cdot \operatorname{Ln}(\mathrm{P} / 101325),
\end{gathered}
$$

上式的相关系数为 1.000 , 相关性很好. 因而可用上式计算 $\mathrm{C}_{60}$ 分子在气相不同温度、不同压 力下的摩尔嫡.

由表 1 可见, $C_{60}$ 分子的气相摩尔等压热容 $C_{p, m}$ 在 $1000 \mathrm{~K}$ 以下, 随温度升高增加得 较快. 但 $1000 \mathrm{~K}$ 以上, 随温度升高增加速度变慢. 当温度达到 $3000 \mathrm{~K}$ 时已接近 $C_{p, m}$ 的极限值 $\left(1488.28 \mathrm{~J} \cdot \mathrm{mol}^{-1} \cdot \mathrm{K}^{-1}\right)$. 为了找到 $\mathrm{C}_{60}$ 分子气相摩尔等压热容与温度之间的简单关系式, 将气相摩尔等压热容 $C_{p, m}$ 对热力学温度 $T(\mathrm{~K})$ 作回归分析, 得下式 $\left(C_{p, m}\right.$ 的单位是 $\left.\mathrm{J} \cdot \mathrm{mol}^{-1} \cdot \mathrm{K}^{-1}\right)$ :

$$
C_{p, m}=-276.855+3.284 \cdot T-2.474 \times 10^{-3} \cdot T^{2}+8.309 \times 10^{-7} \cdot T^{3}-1.026 \times 10^{-10} \cdot T^{4} .
$$

上式的相关系数为 0.9997 , 相关性较好. 因而可用上式来计算不同温度下气相 $\mathrm{C}_{60}$ 分子的摩 尔等压热容. 采用上述同样的方法, 我们也求得 $\mathrm{C}_{60}$ 分子气相等容热容 $C_{v, m}$ 与热力学温度 $T(\mathrm{~K})$ 的关系式如下 $\left(C_{v, m}\right.$ 的单位是 $\left.\mathrm{J} \cdot \mathrm{mol}^{-1} \cdot \mathrm{K}^{-1}\right)$ :

$$
C_{y, m}=-285.169+3.284 \cdot T-2.474 \times 10^{-3} \cdot T^{2}+8.309 \times 10^{-7} \cdot T^{3}-1.026 \times 10^{-10} \cdot T^{4} .
$$
上式可以用来计算不同温度下气相 $\mathrm{C}_{60}$ 分子的摩尔等容热容.

\section{参考文献}

[1] Krätschmer, W., Fostiropoulos, K., Huffman, R., Chem. Phys. Lett., 1990, 170:167.

[2] Jin Yinmin, Cheng Jinlong et al., J. Phys. Chem., 1992, 96(12):5151.

[3] Mathews, C. K., Saibaba, M. et al., J. Phys. Chem., 1992, 96(9): 3566.

[4] Wang, C. Z., Xu, C. H. et al., J. Phys. Chem., 1992, 96(9): 3564. 